\title{
CÁLCULO DA EFICIÊNCIA TÉRMICA DO GLENDON *
}

\author{
Deyve Drengson Carneiro Vieira ${ }^{1}$
} Tiago Luis Oliveira²

\section{Resumo}

O objetivo desse trabalho é identificar a eficiência térmica do glendon de uma empresa do setor siderúrgico mineiro. Tendo em vista que quanto mais eficiente for o equipamento, maior é o aproveitamento da energia fornecida e também menor é o consumo de carvão vegetal no alto-forno, reduzindo o OPEX. Este trabalho foi um estudo de caso baseado na análise de diferentes referências bibliográficas e através de estudos prévios e dados reais. $O$ glendon em estudo apresentou eficiência térmica de 53,1\%. Estudos mais recentes apresentaram eficiência de 67,4\% utilizando chicanas no interior do glendon alterando o fluxo gasoso. O combustível do glendon é o gás do topo do alto-forno recuperado. A energia proveniente da combustão representa $89 \%$ da entrada de energia no regenerador, o que mostra quão importante é uma queima estável do GAF no glendon. Observou-se um desperdício de $45,5 \%$ da energia de combustão que saem pelos gases na chaminé, sendo uma oportunidade futura de estudo para reaproveitamento dessa energia.

Palavras-chave: Altos-fornos; Glendon; Reator.

\section{CALCULATION OF THE THERMAL EFFICIENCY OF GLENDON}

\begin{abstract}
This paperwork focus on identify the thermic efficiency of the Gledon reactor from a steel company in Minas Gerais' State, Brazil. Considering that the more efficient is the equipment, the greater is the utilization of supplied energy and also the less is the consumption of charcoal in the blast furnace, reducing the OPEX. This study was a case study based on the analysis of different references and through previous studies and real data. This Glendon presented thermal efficiency of 53,1\%. More recent studies presented the efficiency of $67,4 \%$ by using baffles inside the glendon which changes the gas flow. The Glendon Fuel is the gas recovered from the top of the furnace. The combustion's energy represents $89 \%$ of the energy input in the regenerator, which demonstrates how important is to have a stable burning of BFA (Blast Furnace Air) in Glendon. It was noticed a waste of $45,5 \%$ of the combustion energy through the gases in the chimmey, which is an opportunity for future study about the reuse of that energy.
\end{abstract}

Keywords: Blast furnace, Glendon; Reactor.

1 Engenheiro Metalurgista, Bacharel, João Monlevade, Minas Gerais, Brasil.

2 Engenheiro Mecânico, Doutorando em Engenharia de Materiais, Redemat- Rede Temática em Engenharia de Materiais-UFOP/UEMG, Ouro Preto, Minas Gerais, Brasil. 


\section{TRODUÇÃO}

O objetivo desse trabalho é identificar a eficiência térmica do regenerador de calor do tipo glendon, realizando seus balanços de massa e energia.

A eficiência térmica do glendon tem grande importância dentro da usina siderúrgica, pois ele é responsável por aquecer o ar soprado no alto-forno, e assim contribuir, na redução de consumo de Biorredutor (carvão vegetal) para a produção do hot metal.

O alto-forno é um equipamento destinado a produção de hot metal em estado líquido a uma temperatura em torno de $1500^{\circ} \mathrm{C}$. Para isso, o alto-forno utiliza como carga metálica (sínter, pelotas e minério granulado) e o combustível sólido (coque ou carvão vegetal) além de fundentes e injeções auxiliares (gás natural, carvão pulverizado ECT.). [7]

Um dos princípios básicos do funcionamento do alto forno é a reação de combustão através da combinação do carbono do combustível com o oxigênio do ar, que é injetado pelas ventaneiras em alta velocidade, gerando calor para aquecimento da carga e gás redutor para o processo de conversão do minério de ferro em ferro metálico. [8]

Os altos-fornos atualmente são responsáveis por aproximadamente $95 \%$ da produção mundial de hot metal (ferro gusa). [8]

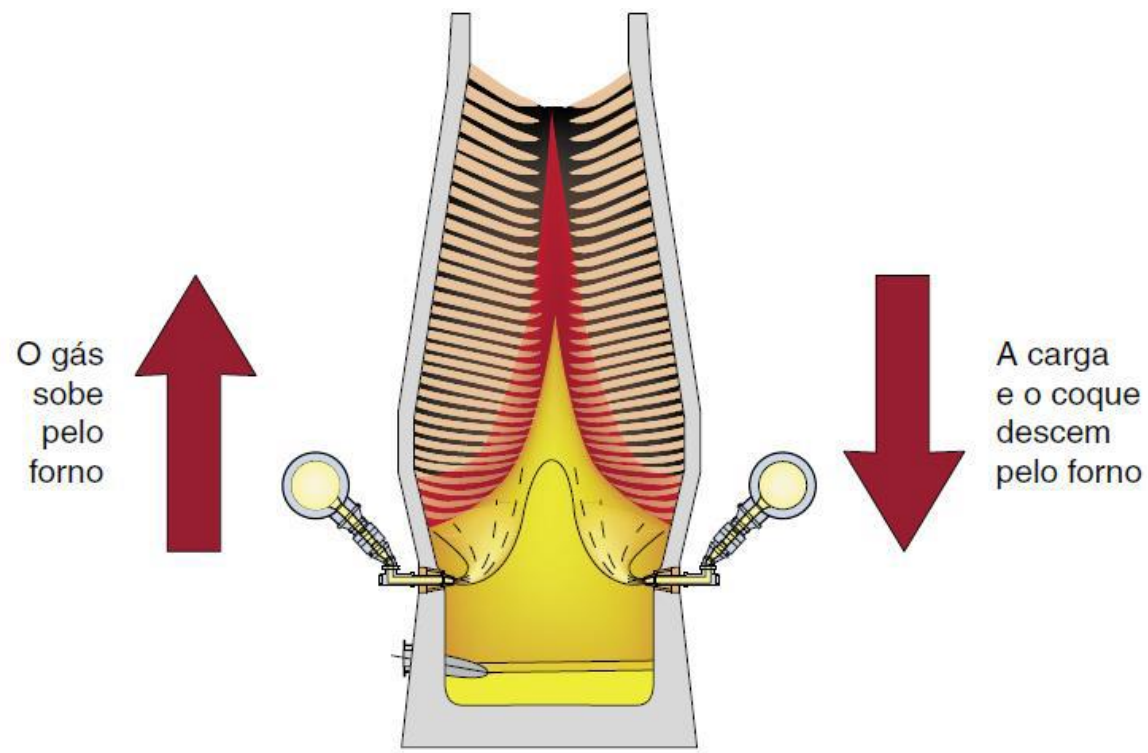

Figura 1. Alto Forno Como um Reator de Contra Corrente [6]

O alto-forno pode ser considerado como um intercambiador de calor e de materiais entre a carga descendente, inicialmente sólida, depois pastosa e mais tarde líquida e o gás redutor que sobe em contracorrente [1]

Apesar de ser baixo o poder calorífico do gás de alto-forno, comparado com os outros gases como os provenientes da aciaria e da coqueria, o mesmo pode ser recuperado e utilizado como combustível no pré-aquecimento do ar de combustão nos regeneradores do próprio alto-forno. [2]

O gás de alto-forno é um combustível de má qualidade, mas é, no entanto, produzido em uma quantidade grande o suficiente para representar uma parte importante do projeto de lei de combustível global da indústria do aço. [5]

Em situações em que há um excesso de GAF em todas as possíveis aplicações para baixa temperatura tem sido satisfeitas, é de interesse de olhar para a 
possibilidade de utilizar o gás para a temperatura mais elevada no processo industrial. [5]

Esses gases são previamente purificados e queimados nos trocadores de calor. [8] A tabela abaixo apresenta varias composições de gases de alto-forno, com seu respectivo poder calorífico: [5]

Tabela 1: Composição e poder calorífico de gases de alto-forno (GAF)

\begin{tabular}{|c|c|c|c|c|c|c|c|c|}
\hline \multirow{2}{*}{ FLAME } & \multicolumn{3}{|c|}{ Blast furnace gas } & \multicolumn{2}{|c|}{ nalysis (z vol.) } & \multirow{2}{*}{$\underset{\left(k c a l / m_{0}^{3}\right)}{\text { LCV }}$} & \multirow{2}{*}{$\begin{array}{c}\text { Stoich. } \\
\text { air } \\
\mathrm{kg} / \mathrm{kg} \text { fuel }\end{array}$} & \multirow{2}{*}{$\begin{array}{l}\text { density } \\
\left(\mathrm{kg} / \mathrm{m}^{3}{ }_{0}\right)\end{array}$} \\
\hline & $\mathrm{CO}_{2}$ & $\mathrm{co}$ & $\mathrm{CH}_{4}$ & $\mathrm{H}_{2}$ & $\mathrm{~N}_{2}$ & & & \\
\hline F 1b & 15.6 & 16.5 & 8.5 & 16.8 & 42.6 & 1662 & 1.845 & 1.124 \\
\hline Elb & 18.3 & 18.6 & 4.2 & 4.2 & 54.7 & 1573 & 1.577 & 1.278 \\
\hline E1c & 18.6 & 19.2 & 3.9 & 3.89 & 54.41 & 1641 & 1.650 & 1.279 \\
\hline E3b & 18.5 & 19.3 & 3.96 & 4.07 & 54.17 & 1595 & 1.594 & 1.280 \\
\hline B11a & 21.4 & 17.2 & 5.0 & 2.83 & 53.57 & 1322 & .917 & 1.347 \\
\hline$B 22 a$ & 22.2 & 16.4 & 4.9 & 3.2 & 53.3 & 999 & .897 & 1.349 \\
\hline B23a & 21.7 & 15.9 & 4.7 & 2.8 & 54.9 & 949 & .855 & 1.351 \\
\hline $\mathrm{B} 33 \mathrm{a}$ & 21.8 & 15.3 & 4.7 & 3.11 & 55.09 & 946 & .853 & 1.348 \\
\hline$B^{k} 22 / 35 a$ & 20.2 & 16.4 & 4.6 & 3.13 & 55.67 & 971 & .874 & 1.337 \\
\hline $\mathrm{B} 23 / 25 \mathrm{a}$ & 20.7 & 16.7 & 4.6 & 2.86 & 55.14 & 973 & .873 & 1.340 \\
\hline B23/25a1 & 21.0 & 16.0 & 4.2 & 2.67 & 56.13 & 913 & .810 & 1.350 \\
\hline B33/25a & 21.5 & 16.3 & 4.3 & 2.89 & 55.01 & 936 & .831 & 1.350 \\
\hline $\mathrm{B} 22 \mathrm{~b}$ & 22.5 & 16.5 & 4.7 & 2.77 & 53.53 & 973 & .866 & 1.357 \\
\hline $3^{\star} 22 / 35 b$ & 22.8 & 17.2 & 4.9 & 2.89 & 52.21 & 1015 & .921 & 1.357 \\
\hline$c^{*} 1 b$ & 19.6 & 18.4 & 3.91 & 3.95 & 54.14 & 993 & $.8 \varepsilon 2$ & 1.327 \\
\hline $\mathrm{ClOb}$ & 19.6 & 18.6 & 4.01 & 3.29 & 54.5 & 991 & $.8 \div 6$ & 1.334 \\
\hline$c^{k}{ }_{100 b}$ & 20.4 & 19.9 & 3.65 & 4.20 & 51.85 & 1025 & $.86:$ & 1.335 \\
\hline C13a & 19.1 & 18.4 & 4.22 & 3.35 & 54.93 & 1004 & .897 & 1.328 \\
\hline $\mathrm{C} 13 \mathrm{~b}$ & 18.5 & 17.8 & 5.04 & 2.72 & 55.94 & 1041 & .946 & 1.327 \\
\hline $\mathrm{C} 13 / 25 \mathrm{~b}$ & 20 & 19.2 & 4.33 & 3.39 & 53.08 & 1039 & .921 & 1.334 \\
\hline $\mathrm{Cl} 13 / 35 \mathrm{~b}$ & 18.6 & 19.4 & 3.8 & 3.42 & 54.78 & 1000 & .885 & 1.326 \\
\hline$c^{k} 1 / 25 b$ & 21.6 & 20.8 & 3.5 & 4.16 & 49.94 & 1036 & .897 & 1.338 \\
\hline$c^{2} 1 / 35 b$ & 19.7 & 19.1 & 3.95 & 4.31 & 52.94 & 1027 & .914 & 1.320 \\
\hline$c^{*} 1 / 50$ & 19.8 & 18.6 & 3.95 & 3.93 & 53.72 & 1002 & .891 & 1.325 \\
\hline$c^{*} 1 / 35 c$ & 19.9 & 18.9 & 4.00 & 4.91 & 52.21 & 1041 & .936 & 1.313 \\
\hline $\mathrm{D}^{\mathrm{k}} 23 \mathrm{~b}$ & 24.0 & 15.6 & 2.5 & 2.97 & 54.93 & 762 & .639 & 1.376 \\
\hline$D^{*} 23 / 25 b$ & 26.0 & 15.1 & 2.8 & 2.84 & 53.26 & 769 & .648 & 1.390 \\
\hline $\mathrm{D}^{\mathrm{k}} 31 \mathrm{~b}$ & 25.2 & 16.1 & 2.8 & 2.59 & 53.31 & 793 & .664 & 1.386 \\
\hline $\mathrm{D}^{\mathrm{k}} 31 / 35 \mathrm{~b}$ & 24.9 & 15.6 & 3.0 & 2.42 & 54.08 & 791 & .668 & 1.385 \\
\hline$D^{k} 11 / 35 b$ & 25.7 & 15.9 & 2.74 & 2.44 & 53.22 & 778 & .648 & 1.392 \\
\hline
\end{tabular}

Se o ar necessário à combustão fosse introduzido pelas ventaneiras do alto forno à temperatura ambiente maior quantidade de Coque ou carvão vegetal seria gasta para gerar calorias para aquecer os gases até as temperaturas de trabalho reinantes na região de queima. [1]
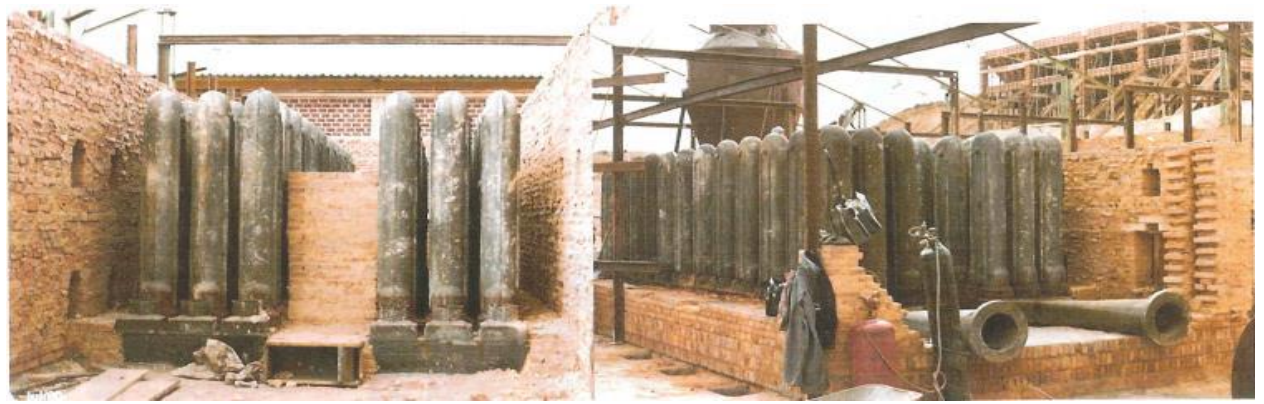

Figura 2. Regenerador Glendon 
Para realizar o aquecimento do ar de combustão nos trocadores de calor é utilizado parte dos gases que saem do topo do alto-forno. Estes gases são devidamente purificados e são queimados nos trocadores de calor. [8]

Segundo Rizzo (2009), o trocador de calor tipo glendon consiste em uma câmara de paredes refratárias, onde ocorre à combustão que consequentemente aquece fileiras de garrafas de ferro fundido, interligadas por canais na parte inferior ou tubulações de aço inox chamadas de serpentinas. O ar frio é soprado escoa por dentro das garrafas ou tubulações e se aquece. [8]

Os Glendons possuem um regime de trabalho contínuo. $O$ ar quente é então conduzido ao anel de vento e injetado no alto-forno através das ventaneiras. [2] Rizzo (2009) afirma que a operação do glendon consiste basicamente em se manter a temperatura do ar de sopro constante. [8]

\subsection{Leis da Termodinâmica}

A primeira lei da termodinamica: "A energia não pode ser criada nem destruida em um sistema de massa constante, mas poder ser convertida de uma forma para outra". [4]

Para o desenvolvimento de balanço de massa é necessário lidar com alguns conceitos: Número de moles, equação dos gases e estequiometria de reações químicas. [4]

O balanço de massa para qualquer processo metalúrgico está baseado na seguinte espressão: [4]

Entrada de massa =Saída de massa +Acumulo de massa

Para o desenvolvimento de balanço de massa é necessário lidar com alguns conceitos: Número de moles, equação dos gases e estequiometria de reações químicas. [4]

A eficiência energética (térmica) de um equipamento pode ser entendida Como a razão entre o resultado obtido e o recurso empregado. No caso dos Glendons, A eficiência energética foi definida. [2]

$\eta=\frac{Q 1}{Q 0} \times 100$

(2)

$\eta=$ Eficiência térmica do glendon; $\mathrm{Q} 1$ = Calor absorvido pelo ar de sopro

Q0 = Calor fornecido pela combustão

\section{MATERIAIS E MÉTODOS}

Este trabalho é um estudo de caso baseado na análise de dados reais de uma empresa do setor siderúrgico e na literatura referente ao assunto de altos-fornos e trocadores de calor.

Na empresa de estudo não se utiliza apenas 1 (um) glendon, mas sim um conjunto formado por 4 (quatro) glendons. Para simplificar foi estudado o glendon número 2, Sua escolha deve-se ao fato de estar em melhores condições físicas e apresentar instrumentos de medidas mais confiáveis. 


\subsection{Premissas}

Na siderúrgica o conjunto é formado por 4 glendons, para simplificação estudou-se o glendon de número 2. Sua escolha deve-se ao fato de estar em melhores condições físicas e apresentar instrumentos de medidas mais confiáveis.

Seguem algumas premissas:

Temperatura ambiente, referência $25^{\circ} \mathrm{C}$; Temperatura do ar e gás de combustão $25^{\circ} \mathrm{C}$; Composição do ar atmosférico constante; A vazão de ar a ser aquecido é 1/4 do total, pois não há medidor de vazão independente.

\subsection{Balanço de massa}

A entrada de massa no glendon acontece de duas formas (a e b) e a saída também ocorre de duas formas ( $c$ e d). A tabela 2 mostra o teor de cada componente dos gases

a) Ar de sopro frio que sofrerá aquecimento para ser soprado no alto-forno;

b) Combustão do GAF com ar atmosférico;

c) Ar de sopro aquecido;

d) Gases da chaminé do glendon.

Tabela 2: composição dos gases utilizados no glendon

\begin{tabular}{cccc}
\hline \multicolumn{2}{c}{ GAF } & \multicolumn{2}{c}{ Ar Atmosférico } \\
\hline \% & \multicolumn{3}{c}{$\%$} \\
\hline $\mathrm{O} 2$ & 0,74 & $\mathrm{~N} 2$ & 77,23 \\
\hline $\mathrm{CH} 4$ & 2,02 & $\mathrm{O} 2$ & 20,53 \\
\hline $\mathrm{H} 2$ & 4,77 & 2,24 & 2,24 \\
\hline $\mathrm{N} 2$ & 51,53 & - & - \\
\hline $\mathrm{CO}$ & 24,52 & - & - \\
\hline $\mathrm{CO} 2$ & 16,36 & - & - \\
\hline
\end{tabular}

Como já se sabe o volume parcial de cada composto o cálculo de mol de cada composto se da pela equação:

$n i=V(N m 3) \times 1000 \times(\%$ i $) 100 \times 122.4$

$n i=$ número de mol do elemento/composto;

$\mathrm{V}=$ volume em $\mathrm{Nm}^{3}$;

$\% \mathrm{i}=$ percentual em volume do elemento composto no gás.

Conhecendo a quantidade em mol de água, pode calcular o volume nas condições normais de temperatura e pressão:

$1000 \times V(N m 3) V(N m 3) H 2 O=n H 2 O \times V H 2 O$

Tabela 4: Vazão dos glendons

\begin{tabular}{cccccc}
\hline Entrada & unidade & Mês 1 & Mês 2 & Mês 3 & Média \\
\hline Vazão Total & $\mathrm{Nm}^{3} / \mathrm{h}$ & 20737 & 21850 & 20046 & 20878 \\
\hline Vazão glendon 2 & $\mathrm{Nm}^{3} / \mathrm{h}$ & 5184 & 5462 & 5011 & 5219 \\
\hline Vazão glendon 2 & $\mathrm{Nm}^{3} / \mathrm{s}$ & Parede & 1,52 & 1,39 & 1,45 \\
\hline
\end{tabular}


A vazão da mistura de combustão Ar/GAF não é medida no processo. Sendo assim, não é possível ser feito balanço de massa diretamente.

Então foi realizado o balanço de massa indiretamente. Foi realizado o balanço de massa para queima de $1 \mathrm{Nm}^{3}$ de GAF.

\subsection{Balanço de energia}

Para o cálculo de energia utilizou-se dados conforme tabela 5:

Tabela 5: Entradas e saídas de calor

\begin{tabular}{cc}
\hline Entrada de Calor & Saída de calor \\
\hline $\begin{array}{c}\text { Calor de reação ar atm com } \\
\text { GAF }\end{array}$ & $\begin{array}{c}\text { Aquecimento do ar de } \\
\text { sopro }\end{array}$ \\
\hline $\begin{array}{c}\text { Calor contido no ar de } \\
\text { sopro frio }\end{array}$ & $\begin{array}{c}\text { Perda pelas paredes } \\
\text { e teto }\end{array}$ \\
\hline- & $\begin{array}{c}\text { Perdas pelos gases } \\
\text { de chaminé }\end{array}$ \\
\hline
\end{tabular}

A entrada de energia se dá pelo calor contido no ar de sopro frio e reação de combustão ar/GAF.

A energia contida no ar de sopro pode ser calculada com a expressão abaixo:

$(H T-H 298) e=n e(A T+B \times 10-3 \times T 2+c t \times 105+D)$

Essa temperatura do ar de sopro frio é medida mas não é registrada. Para formação de dados foi realizado um acompanhamento aleatório para calcular a média dessa temperatura durante 10 dias, Conforme tabela 6.

Tabela 6: Temperatura de ar de sopro frio

\begin{tabular}{cccccccccccc}
\hline \multicolumn{10}{c}{ Temperatura de ar de sopro na entrada do glendon } \\
\hline Dia & $\mathbf{1}$ & $\mathbf{2}$ & $\mathbf{3}$ & $\mathbf{4}$ & $\mathbf{5}$ & $\mathbf{6}$ & $\mathbf{7}$ & $\mathbf{8}$ & $\mathbf{9}$ & $\mathbf{1 0}$ & Média \\
\hline${ }^{\circ} \mathrm{C}$ & 134 & 131 & 138 & 135 & 126 & 129 & 140 & 131 & 119 & 138 & 132 \\
\hline
\end{tabular}

A energia de combustão de GAF/Ar foi calculada através das entalpias das reações químicas a seguir $(6,7,8,9)$.

$$
\begin{aligned}
& \Delta H^{\circ} \mathrm{R}=n e\left(\Delta H^{\circ} 298 \mathrm{P}\right)-n e\left(\Delta H^{\circ} 298 \mathrm{r}\right) \\
& \mathrm{H}_{2}+\mathrm{O}_{2} \rightarrow \mathrm{H}_{2} \mathrm{O}-\Delta \mathrm{H}^{\circ} 298=17,99 \mathrm{KCal} / \mathrm{Mol} \\
& \mathrm{CO}+\mathrm{CO}_{2} \rightarrow \mathrm{CO}_{2}-\Delta \mathrm{H}^{\circ} 298=67,634 \mathrm{KCal} / \mathrm{Mol} \\
& \mathrm{CH} 4+2 \mathrm{O}_{2} \rightarrow \mathrm{CO}_{2}+2 \mathrm{H}_{2} \mathrm{O}-\Delta \mathrm{H}^{\circ} 298=299 \mathrm{KCal} / \mathrm{Mol}
\end{aligned}
$$

A saída de energia se da por aquecimento do ar de sopro, pelos gases que saem pela chaminé e perda pelas paredes e perdas pelo teto.

Foi realizado um levantamento utilizando dados históricos para determinar a temperatura média em que o ar de sopro é aquecido, Conforme tabela 7.

Tabela 7: Temperatura do ar de sopro aquecido

$$
\text { Temperatura do ar de sopro aquecido }
$$

\begin{tabular}{ccccc}
\hline Temperatura & Mês 1 & Mês 2 & Mês 3 & Média \\
\hline${ }^{\circ} \mathrm{C}$ & 671 & 663 & 672 & 672 \\
\hline
\end{tabular}


A temperatura dos gás que saem da chaminé são medidas mas não são registradas. Para criação da base de dados foram realizadas amostragens de temperatura durante 10 do mês 3 . Foi tomado o cuidado em não coletar dados em dias em que ocorreram anomalias no processo (tabela 8).

Tabela 8: temperatura do gás da chaminé do glendon

\begin{tabular}{cccccccccccc}
\hline \multicolumn{10}{c}{ Temperatura do gás da chaminé } \\
\hline Dia & $\mathbf{1}$ & $\mathbf{2}$ & $\mathbf{3}$ & $\mathbf{4}$ & $\mathbf{5}$ & $\mathbf{6}$ & $\mathbf{7}$ & $\mathbf{8}$ & $\mathbf{9}$ & $\mathbf{1 0}$ & Média \\
\hline${ }^{\circ} \mathrm{C}$ & 475 & 486 & 478 & 430 & 484 & 470 & 397 & 435 & 397 & 458 & 451 \\
\hline
\end{tabular}

Não foi possível calcular as perdas térmicas pelas paredes e tetos. Para calcular as perdas seriam necessárias informações sobre o material de construção do glendon tais como, condutividade térmica.

Descrever brevemente os equipamentos e os procedimentos utilizados, assim como a literatura e os métodos estatísticos empregados, quando for o caso.

\section{RESULTADOS E DISCUSSÃO}

O glendon em estudo apresentou eficiência térmica de $53,1 \%$. A perda pelos gases da chaminé foi de $45,5 \%$. Como proposto, a perda pelas paredes e teto aproximadamente $2,2 \%$ (figura 3). É clara uma grande perda de energia pelos gases de chaminé, se aproximando a eficiência térmica do glendon.

\section{Saidas de energia de combustão}

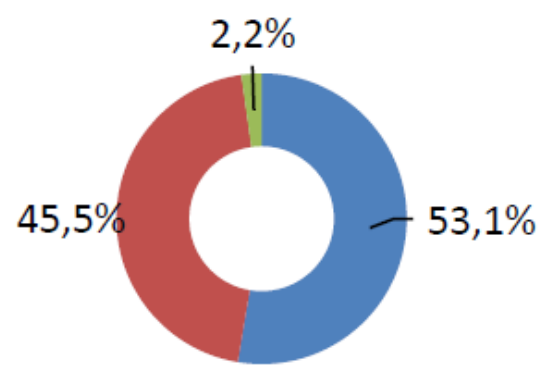

Ar de sopro achaminé $\square$ Paredes e teto

Figura 3. Saída de energia do Glendon

O glendon em estudo apresentou eficiência próxima do encontrado na literatura $51,3 \%$. Eficiência energética dos Glendons é da ordem $59,1 \%$. [2]

Após alguns trabalhos realizados com instalação de chicanas Assunção conseguiu elevar a eficiência para $67,4 \%$. A inclusão das chicanas se mostrou mais efetiva do que a redução da área de passagem dos gases da combustão, e promoveu um aumento na eficiência energética do Glendons de $59,1 \%$ para $67,4 \%$. O objetivo foi fazer com que o fluxo dos gases da combustão fosse cruzado ao fluxo de ar no interior das serpentinas e a turbulência do escoamento externo fosse aumentada, aumentando assim a troca de calor (Figura 4). [2] 


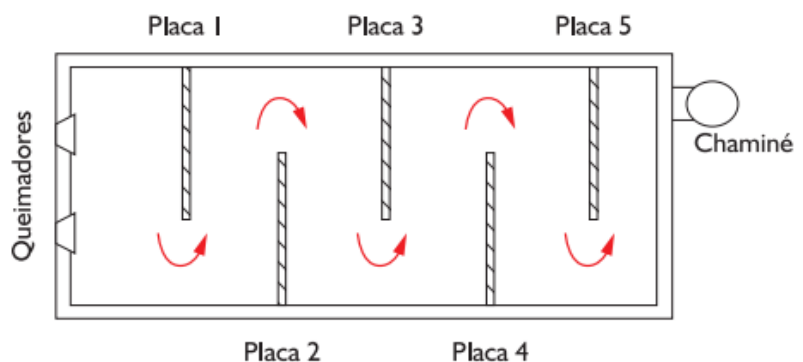

Figura 4. Esquema das chicanas no interior do glendon

De acordo com os cálculos de balanço de massa, para uma queima completa do GAF a relação de ar/GAF é 0,84 . Para garantia de uma queima completa é utilizado um relação com excesso de 02 1,1 (figura 5).

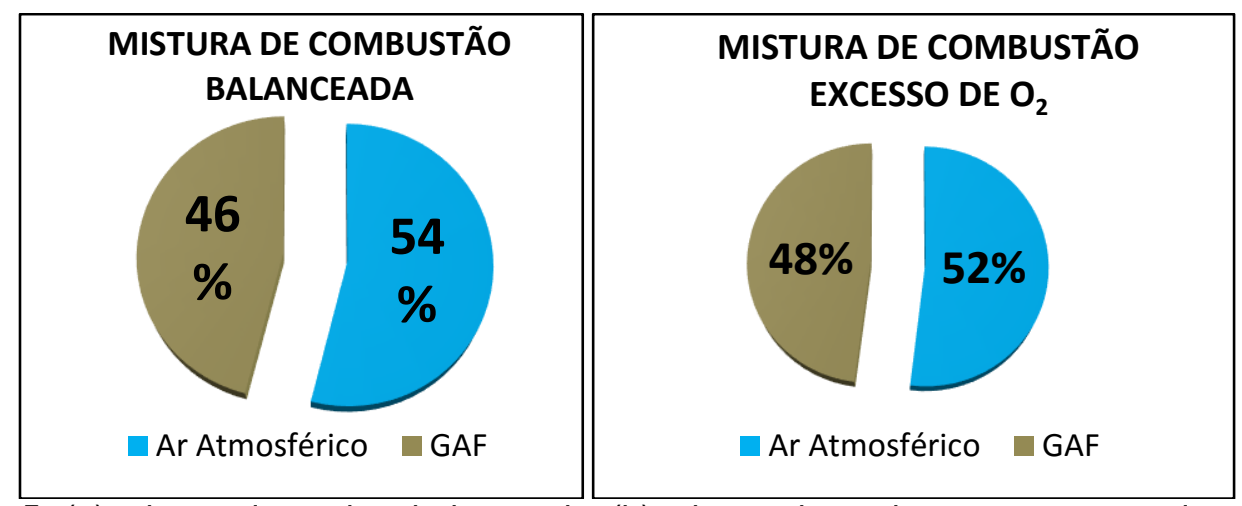

Figura 5: (a) mistura de queima balanceada, (b) mistura de queima com excesso de oxigênio

A energia proveniente da combustão representa $89 \%$ da entrada de energia no regenerador, o que mostra quão importante é uma boa queima do GAF (figuras 6 ).
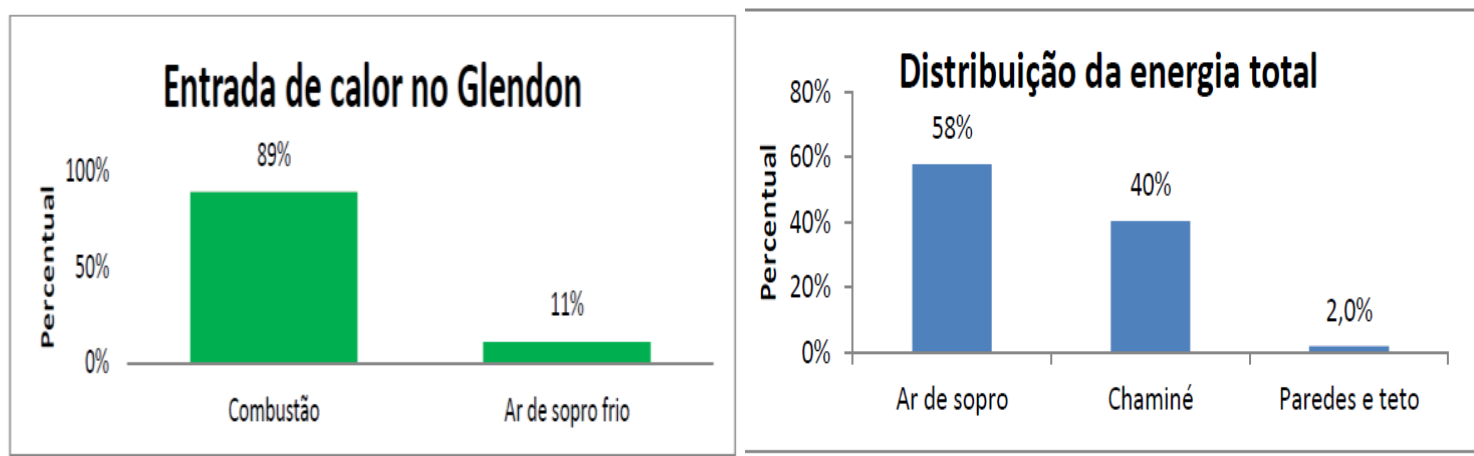

Figura 6: (a) Entrada de calor no glendon, (b) Saída da energia do glendon

Tendo em vista de outro trabalho relacionado, é verificado que as perdas térmicas pelo teto e paredes são pequena. Segundo (ASSUNÇÃO, 2006) a perda térmica pelas paredes e teto representa cerca de $1 \%$ da energia liberada pela combustão do GAF.[2]

Visando que o modelo de equipamento tem algumas diferenças e que nesse trabalho não foi calculado. Esse tipo de perda foi considerada em até $2,6 \%$ da energia produzida pelo GAF. (Tabela 9). 
Tabela 9. Entrada e Saída De Calor Do Glendon

\begin{tabular}{cccc}
\hline Entrada & Kcal & Saída & Kcal \\
\hline $\begin{array}{c}\text { Ar de sopro } \\
\text { Frio }\end{array}$ & 62 & $\begin{array}{c}\text { Ar de sopro } \\
\text { aquecido }\end{array}$ & 331 \\
\hline Combustão & 505 & Chaminé & 230 \\
\hline- & - & Parede & 11 \\
\hline Total & 568 & & 572 \\
\hline
\end{tabular}

\section{CONCLUSÃO}

A realização do cálculo da eficiência térmica do glendon foi importante para conhecer o verdadeiro estado de eficiência térmica do conjunto de glendons da empresa em estudo. Sendo que em mais de 19 anos em operação do equipamento nunca foi realizado um estudo dessa amplitude.

glendon em estudo apresentou boa eficiência térmica 53,1\%. Do total de energia que entra no regenerador $58 \%$ é absorvida pelo ar de sopro, sendo que $89 \%$ desta energia sendo proveniente da queima do GAF.

Para que fosse possível chegar ao resultado foi de grande importância conhecer, através de uma relação matemática, a quantidade de ar atmosférico necessário para que seja possível a queima desejada do GAF, que para a composição química do gás em estudo foi de $0,84 \mathrm{ar} / \mathrm{GAF}$.

A bibliografia sobre glendon é pequena e de difícil acesso. O regenerador é um equipamento que tem poucos instrumentos de controle e acompanhamento o que dificulta a obtenção de dados precisos e confiáveis, o que demanda um esforço maior na coleta e tratamento dos dados obtidos.

Como a energia de queima é de extrema importância para o boa eficiência térmica do glendon, a automatização das válvulas que regulam a relação ar/GAF é uma condição que levará à um avanço no sentido de aproveitar melhor o potencial energético do GAF.

Em seu trabalho (ASSUNÇÃO 2009) afirma que, com um aumento real na temperatura de sobro de $135^{\circ} \mathrm{C}$. [2]

Para trabalho futuro o estudo de viabilidade econômica da implantação das chicanas abre uma grande oportunidade de melhoria da eficiência energética do glendon.

Observou-se uma grande perda de energia pelos gases da chaminé, o aprofundamento em estudos em reaproveitamento desses gases na secagem e tratamento térmico em minérios é outra oportunidade de avanços.

\section{Agradecimentos}

Meus Agradecimentos vão para: Professor Dr. Tiago Oliveira pelo apoio, confiança e orientação nesse trabalho desde o início; Engenheiro metalurgista Cristhian Luiz Gomes pelo suporte técnico e prático; FaEnge e Departamento de Engenharia Metalurgia pela parceria firmada academia - indústria, sem a qual não seria possível a realização do projeto; A empresa em questão empresa por abrir as portas para realização dos trabalhos; Aos departamentos de Engenharia Ambiental, Minas e Civil por terem se mobilizado em conjunto as atividades do projeto; Professores Breno Eustáquio e Marcelo Alves Fonseca pelo apoio com a metodologia. A coordenação de pesquisa da Faenge por acreditar no projeto; Sara Oliveira pelo apoio. 


\section{REFERÊNCIAS}

1 ARAUJO, Luiz Antonio de, Manual de Siderurgia (2ª Ed., Vol.1). São Paulo: Arte \& Ciência, 2005.

2 ASSUNÇÃO, Charles Sóstenes. Glendon Siderúrgico: Análise Termodinâmica e Modelagem Matemática Simplificada. Dissertação apresentada ao programa de pósgraduação Stricto Sensu de Engenharia Metalúrgica da Universidade Federal de Minas Gerais (UFMG) em 2006. Belo Horizonte: UFMG, 2006.

3 ASSUNÇÃO, C. S. Redução do consumo de oxigênio em um alto-forno a partir do aumento da eficiência energética dos aquecedores de ar In: ENCONTRO DE PRODUTORES E CONSUMIDORES DE GASES INDUSTRIAIS, 25., SEMINÁRIO DE BALANÇOS ENERGÉTICOS GLOBAIS E UTILIDADES, 2009, São Paulo. Anais. São Paulo: ABM, 2009.

4 CASTRO, Luiz Fernando Andrade de; TAVARES, Roberto Parreiras; MORELATO, Anderson Peter; Injeção de Carvão Pulverizado nas Ventaneiras do Alto-Forno. UFMG, Belo Horizonte, 1997.

5 Commission Of The European Communities. The use of blast-furnace gas as a fuel in high-temperatura furnaces of the steel industry. EUR 1979

6 GEERDES, Geerdes et al. Práticas Modernas para Operação de Alto Forno. Cidade de IJmuiden, Outubro de 2004.

7 MOURÃO et al. Introdução à Siderurgia. ABM, São Paulo, 2007.

8 RIZZO, Ernandes Marcos da Silveira. Processo de Fabricação de Ferro-Gusa em AltoForno. ABM, São Paulo, 2009. 